\title{
Reviewer Acknowledgements for Vol. 6, No. 4, December 2018
}

Journal of Agricultural Studies would like to acknowledge the following reviewers for their assistance with peer review of manuscripts for this issue. Many authors, regardless of whether JAS publishes their work, appreciate the helpful feedback provided by the reviewers. Their comments and suggestions were of great help to the authors in improving the quality of their papers. Each of the reviewers listed below returned at least one review for this issue.

\section{Reviewers for Volume 6, Number 4}

Abhishek A. Cukkemane, Bijasu Agri Research Laboratory LLP, India

Aftab Alam, Edenworks Inc. New York, USA

Anil Kumar Matta, Vaddeswaram, Guntur dst, India

Babak Mohammadi, University of Tehran, Iran

Chenlin Hu, The Ohio State University, USA

Eben von Well, Agricultural Research Council, South Africa

Eliana Mariela Werbin, National University of Cordoba, Argentinian

Ernest Baafi, CSIR-Crops Research Institute, Ghana

Il Rae Rho, Gyeongsang National University, South Korea

Jiban Shrestha, Nepal Agricultural Research Council, Nepal

Sait Engindeniz, Ege University Faculty of Agriculture, Turkey

Selmi Houc, University of Jandouba, Tunisia

Servet Aras, Bozok University, Turkey

Somaia Alkhair, Alzaeim Alazhari University, Saudi Arabia

Soto Caro Ariel Reinaldo, Universidad de Concepción, Chile

Zakaria Fouad Abdallah, National Research Centre, Egypt

Richard Williams,

Editor

Journal of Agricultural Studies

Macrothink Institute

5348 Vegas Dr.\#825

Las Vegas, Nevada 89108

United States

Phone: 1-702-953-1852 ext.521

Fax: 1-702-420-2900

Email: jas@macrothink.org

URL: http://jas.macrothink.org 